\title{
Bone Resorption Rate of Moved and Fixed Teeth during Alveolar Bone Remodeling by Orthodontic Treatment*
}

\author{
Hajime MORIKAWA**, Seiki YAMANAMI***, \\ Morimasa NISHIHIRA***, Katsuyuki YAMAMOTO***, \\ Yoshiaki SATOH ${ }^{* * * *}$, Hiroyuki ISHIKAWA**** \\ and Shinji NAKAMURA****
}

\begin{abstract}
Orthodontic treatment is based on the remodeling of the alveolar bone in response to externally applied orthodontic force to a tooth. The rate of bone resorption is, therefore, one of the essential factors for understanding tooth movement during orthodontic treatment. There is the basic concept of differential force in orthodontics that the application of an optimal force enables the canine to be moved without moving the molar that is used as an anchor for retracting the canine. In this paper, the resorption rates of the alveolar bone around the molar and the canine were estimated from the clinical measurements of tooth movement and the stress analyses of tooth roots using a 3-D finite element model. The model consisted of an orthodontic spring, the canine, the second premolar, and the first and second molars. The bone resorption rate to a unit stress at the molar was found to be about $0.5 \mathrm{micron} /(\mathrm{kPa} \cdot$ day $)$ and almost the same as that of the canine. This result suggests that the concept of differential force can be explained simply by differences in the geometry of the roots of moved and fixed teeth.
\end{abstract}

Key Words: Biomechanics, Finite Element Method, Shape Measurement, Stress Analysis, Bone Remodeling, Orthodontics, Alveolar Bone, Tooth Movement, Bone Resorption Rate

\section{Introduction}

The purpose of orthodontic treatment is to rearrange irregular teeth and to correct malocclusions, which may induce temporomandibular arthrosis and other masticatory diseases. The development of disordered teeth is mainly due to insufficient growth of

* Received 12th April, 1999. Japanese original: Trans. Jpn. Soc. Mech., Vol.64, No.626, C(1998), p. 38883896 (Received 16th October, 1997)

** Department of Control and Information Engineering, Asahikawa National College of Technology, Shunko-dai 2-2, Asahikawa, Hokkaido 071-8142, Japan. E-mail : morikawa@bmsun.asahikawa-nct.ac.jp

*** Division of Biomedical System Engineering, Graduate School of Engineering, Hokkaido University, North 13 West 8, Kita-ku, Sapporo, Hokkaido 0608628, Japan

**** Department of Orthodontics, School of Dentistry, Hokkaido University, North 13 West 6, Kita-ku, Sapporo, Hokkaido 060-8586, Japan the jawbone not allowing space for the correct positioning of teeth on the dental arch. In clinical orthodontics, a tooth that has little influence on the masticatory system, such as the first premolar, is usually extracted before treatment, and then the other teeth are rearranged to achieve an ideal dental arch. Tooth movement during orthodontic treatment is induced by the remodeling of the alveolar bones, which support the teeth. Namely, when orthodontic force is applied to the crown of a tooth, the force is transferred to the tooth root and causes changes in the mechanical environment of the alveolar bone. Bone resorption and bone apposition are then caused by osteoclasts in compressive stress regions and osteoblasts in tensile stress regions, respectively, thereby resulting in tooth movement. The relationship between orthodontic force applied to the crown and tooth movement at the crown is a matter of primary concern to clinical orthodontists. However, the causal relation of biomechanical responses to mechanical 
stress or strain around a tooth root is also crucially important and needs to be clarified ${ }^{(1)}$.

With regard to stress distributions around tooth roots during orthodontic treatment, several studies using a finite element method have been reported ${ }^{(2)-(4)}$. However, deformation of the alveolar bone during treatment, namely the resorption and apposition of the alveolar bone, has only been modeled by a twodimensional analysis ${ }^{(5)}$. In addition, there are almost no studies on the deformation of the alveolar bone based on clinical measurements. The authors proposed a method of estimating the alveolar bone resorption rate to a unit stress on the basis of stress analyses around the tooth and clinical measurements of tooth movement; the stress distributions were analyzed using a three-dimensional (3-D) finite element method, and the movement of patient teeth were measured three-dimensionally during orthodontic treatment. The bone resorption rate at the canine was estimated on the basis of these measurements ${ }^{(6)}$.

A tooth that is moved in the distal direction, such as a canine, is generally called a moved tooth and it is retracted by the molar, which is called the fixed tooth. It is believed that only a moved tooth can be efficiently retracted by a fixed tooth without causing it to move when an optimal force is applied to the teeth. In other words, the corresponding optimal stress around a moved tooth root induces maximum alveolar bone resorption, whereas the stress less than the threshold causes little resorption, thus allowing a specific tooth to be moved selectively ${ }^{(1)}$. This concept is known as differential force and it is widely accepted as valid in orthodontics. However, this concept has not yet been verified quantitatively.

In this study, we applied the above-mentioned method of estimating bone resorption rates to molars during orthodontic treatment and estimated the alveolar bone resorption rate to a unit stress around a fixed tooth. A 3-D finite element model of teeth including the molars and the canine was constructed, and stress distributions around these teeth were calculated. Alveolar bone resorption around the first molar and the canine were also obtained from the clinical measurement of 3-D movement. The bone resorption rates to a unit stress for fixed and moved teeth were then obtained and compared.

\section{Alveolar Bone Resorption Rate}

We have reported the estimation method of alveolar bone resorption rate to a unit stress in a previous paper ${ }^{(6)}$. In this section, we describe the 3-D finite element model of teeth constructed for this study and briefly mention the estimation method of the alveolar bone resorption.
Table 1 Clinical orthodontic treatment conditions used in our analyses

\begin{tabular}{|c|c|c|c|}
\hline Tooth & Appliance & $\begin{array}{c}\text { Force } \\
\mathrm{N}\end{array}$ & Number of teeth \\
\hline \multirow{3}{*}{ Maxillary canine } & Simple bop wire & 1.47 & 4 \\
\hline & Power chain & 1.47 & 4 \\
\hline & Power chain & 2.94 & 6 \\
\hline \multirow{3}{*}{ Maxillary first molar } & Simple loop wire & 1.47 & 5 \\
\hline & Power chain & 1.47 & 2 \\
\hline & Power chain & 2.94 & 4 \\
\hline
\end{tabular}

Table 2 Mechanical properties of finite element models

\begin{tabular}{ccc}
\hline Materials & Young's modulus & Poisson's ratio \\
\hline Simple loop wire & $15.3 \mathrm{GPa}$ & 0.3 \\
Bracket & $20 \mathrm{GPa}$ & 0.3 \\
Tooth & $20 \mathrm{GPa}$ & 0.3 \\
Periodontal ligament (PDL) & $0.68 \mathrm{MPa}$ & 0.49 \\
\hline
\end{tabular}

\subsection{Finite element analyses of teeth}

Force and stress analyses are conducted on a workstation (Apollo Domain, Hewlett-Packard) using a 3-D general-purpose finite element analysis package (NISA II/DISPLAY III, Engineering Mechanics Research).

2.1.1 Analyses of orthodontic force systems Orthodontic treatment conditions of the subjects analyzed in this study are shown in Table 1. Although a face bow (an extraoral appliance used to prevent the movement of maxillary molars) was also used at night in some subjects, for sake of simplicity the effect of this appliance was not taken into account in this study.

The orthodontic appliances used in the treatments were Bull loop wires and power chains. The former are simple open-loop wires made of an alloy, and the latter are elastic chains made of synthetic rubber. The shapes of the Bull loop wires were fed into a computer with a scanner. Finite element models of the Bull loop wires (hereafter referred to as wire models) were constructed with $3-\mathrm{D}$ beam elements. Table 2 shows the mechanical properties of the wire, which were determined from a previous experiment $^{(7)}$ and used in the wire models.

In clinical orthodontic treatment, gable and antirotation bends are usually incorporated on a wire in order to set a proper moment so that the tooth is moved without tipping or rotation. These bends were also taken into account in each wire model according to the treatment conditions, because the magnitude and direction of the force produced by the bends differ greatly. Figure 1 shows an example of a wire model with both gable and anti-rotation bends set at an angle of 20 degrees. When analyzing a wire model incorporating bends, forced displacements were 


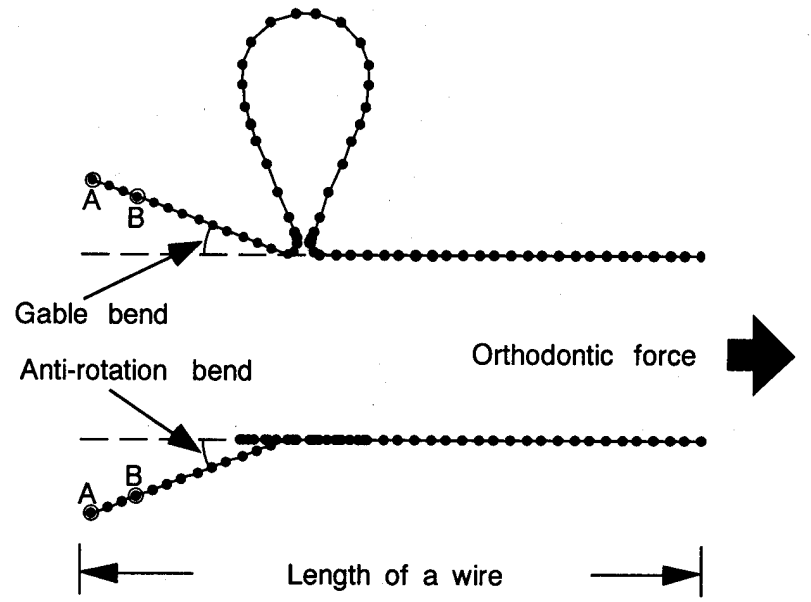

Fig. 1 A finite element model of an analyzed simple loop wire with gable and anti-rotation bends

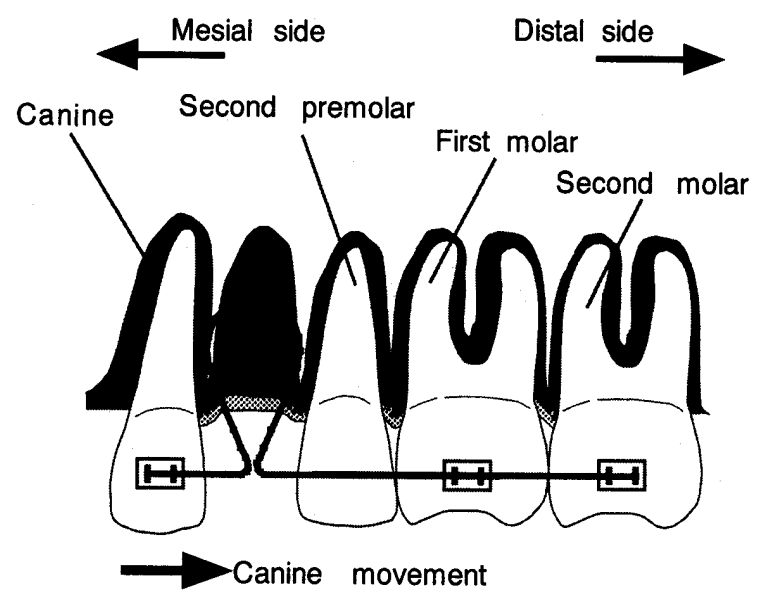

Fig. 2 Schematic diagram of a typical orthodontic treatment

applied to the wire so as to fix it to a bracket, thereby resulting in apparent bends of about 0 degree. A horizontal retraction force was then applied to the right end of the model. Resulting forces transferred to the bracket at points A and B shown in Fig. 1 were obtained by large-deformation analysis.

Since an elastic power chain exerts orthodontic force to the bracket as a point force, finite element analysis of this appliance is not needed. However, after the initial activation of this appliance there is a marked and rapid decrease in the force exerted. This effect was taken into account on the basis of the experimentally obtained results ${ }^{(6)}$; i.e., when the initial orthodontic forces were set at $2.94 \mathrm{~N}$ (300 gf) and $1.47 \mathrm{~N}$ (150 gf), effective forces of $1.96 \mathrm{~N}$ (200 gf) and $1.08 \mathrm{~N}$ (110 gf), respectively, were used in the analyses.

2.1.2 Stress analyses around tooth roots As mentioned above, the first premolar is usually extracted before beginning treatment in order to make space in the dental arch. Labia-side teeth such as the canines are then retracted in the distal direction by
Table 3 Geometric dimensions of teeth

\begin{tabular}{ccccc}
\hline & $\begin{array}{c}\text { Total } \\
\text { Length } \\
\mathrm{mm}\end{array}$ & $\begin{array}{c}\text { Length } \\
\text { of crown } \\
\mathrm{mm}\end{array}$ & $\begin{array}{c}\text { Length } \\
\text { of root } \\
\mathrm{mm}\end{array}$ & $\begin{array}{c}\text { Width } \\
\text { of crown } \\
\mathrm{mm}\end{array}$ \\
\hline Canine & 22 & 7 & 15 & $8.7 \times 10.1$ \\
Second premolar & 19 & 5 & 14 & $7.0 \times 10.3$ \\
First molar & 18 & 5 & 13 & $10.5 \times 12.1$ \\
Second molar & 16 & 5 & 11 & $9.4 \times 11.4$ \\
\hline
\end{tabular}

Table 4 Number of elements for each tooth model

\begin{tabular}{ccccc}
\hline & Tooth & PDL & Bracket & Total \\
\hline Canine & 352 & 960 & 4 & 1316 \\
Second premolar & 304 & 896 & 0 & 1200 \\
First molar & 699 & 2252 & 24 & 2975 \\
Second molar & 643 & 1964 & 32 & 2639 \\
\hline
\end{tabular}

the molars which act as anchors (Fig. 2). In this study, we focused on this typical form of orthodontic treatment.

The 3-D finite element model consisted of the canine, second premolar, first molar and second molar. The teeth were modeled from a set of maxillary anatomical human tooth models as follows. Silicone rubber replicas of each tooth model were made and sliced at intervals of $1 \mathrm{~mm}$ to obtain crosssectional profiles. Enlarged photocopies of the profiles were entered into a computer using a digitizer, and the 3-D finite element model was constructed by piling up these profiles. Dimensions of each tooth are listed in Table 3. The finite element model also included the periodontal ligament (PDL), which is a fibrous soft tissue of about $0.2 \mathrm{~mm}$ that surrounds the tooth root. The PDL was modeled in four layers. Although the external surface of the PDL is connected to the alveolar bone, it was not included in the model but simplified by introducing the following boundary condition. All the nodes on the external surface of the PDL were fixed, treating the bone as a rigid body because the alveolar bone is sufficiently harder than the PDL. We also considered force transfer through contact of the crowns of the fixed teeth. In the model, the crowns that came into contact with each other were connected with a rigid-link element, which consisted of two nodes and which could allow the transfer of displacement from one node to the other. Thus, orthodontic force was also transferred to teeth without an orthodontic appliance. Orthodontic forces via the appliance were applied to the canine and the first or the second molar in the model, according to the treatment conditions. Bracket elements for loading were also added on the crown of the teeth.

The model was constructed using 3-D eight-node solid elements. The number of elements for each tooth model is shown in Table 4. It was assumed that the mechanical properties of all components in the 


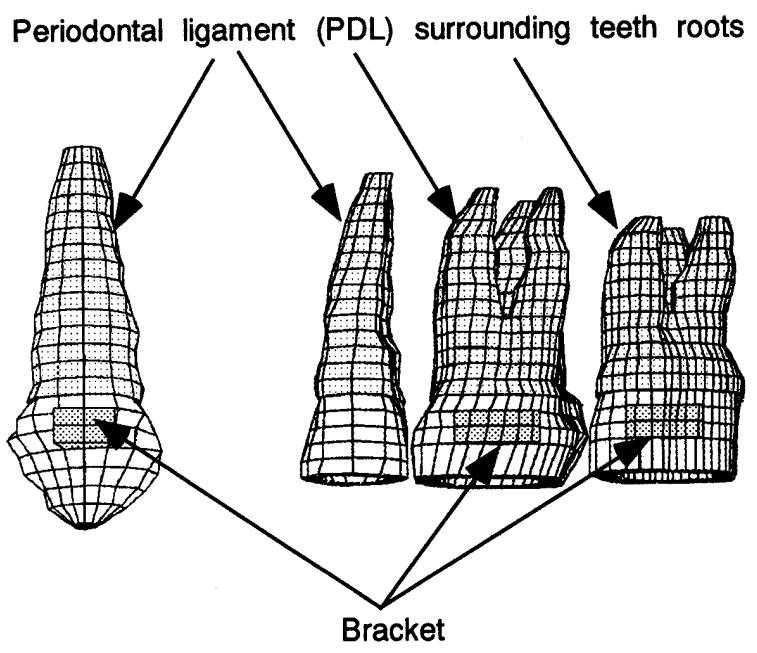

Canine

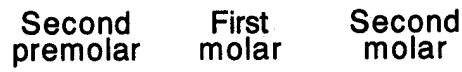

Fig. 3 A finite element model for canine, second premolar, first molar, and second molar. Each tooth model consists of tooth, periodontal ligament (PDL), and a bracket

model were linear and isotropic; the values selected from literature ${ }^{(2)-(4)}$ are shown in Table 2 and were used for the analysis.

The finite element model constructed through the above-mentioned procedures is shown in Fig. 3. Orthodontic forces applied to the brackets were obtained from finite element analyses of the wire models described in the previous section, and the stress distribution on the outer surface of the PDL was then analyzed. We examined the distributions of the minimum principal stress because it has been reported that the appearance of osteoclasts is closely related to the principal stress ${ }^{(2),(8),(9)}$.

\subsection{Estimation of alveolar bone resorption}

Estimation of the alveolar bone resorption was based on 3-D measurements of dental casts taken from patients during orthodontic treatment. The method used is described in a previous paper ${ }^{(6)}$. Briefly, dental plaster casts were taken from the patients at intervals of about one month during treatment. Three-dimensional profiles of the casts were measured by an optical, 3-D measurement system, which we developed, and profile data were fed into a computer. Three-dimensional tooth movement was determined by a $3-D$ registration technique. With this technique, we can compare the tooth profiles at the beginning of treatment and those at a certain stage of treatment and calculate the values of six variables (three translations and three rotations) representing the 3-D movements of each tooth ${ }^{(10)}$.

If we are able to obtain 3-D profile of the whole tooth, including the root, the 3-D movement of a tooth root can be calculated from the estimated movements

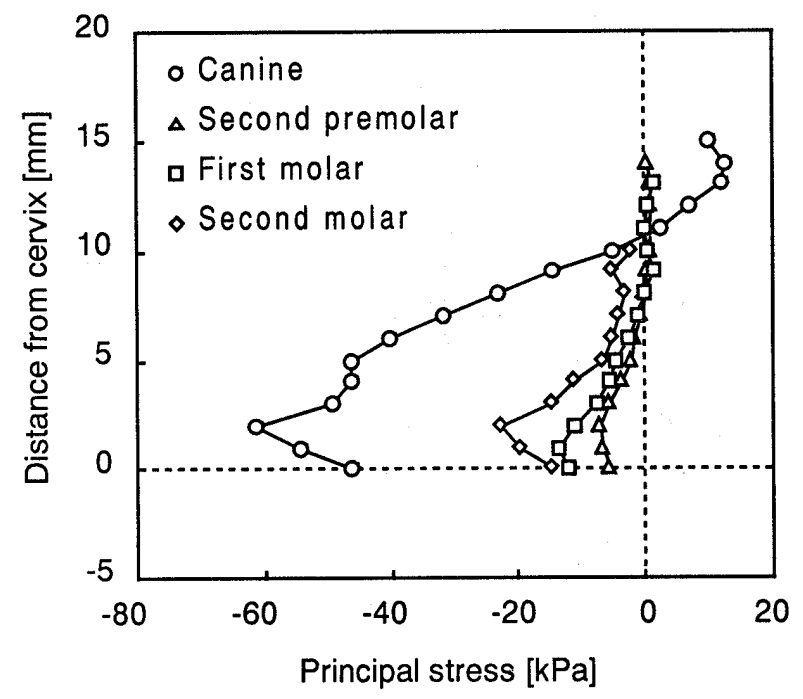

Fig. 4 An example of calculated principal stress distributions on external surface of the PDL

of the crown. However, it is difficult, clinically, to obtain the 3-D profile of a tooth root. Therefore, the tooth models for the finite element analysis were also used to estimate the root movements. The 3-D movements at the crown were applied to the tooth models by coordinate transformation, and the movements of the nodes on the outer surface elements of the PDL were calculated. Alveolar bone resorptions around the root were estimated from the displacement of the nodes in the distal or mesial direction ${ }^{(6)}$.

\section{Results}

\subsection{Stress around a tooth root}

In some schoolchildren under treatment, the second molar has not erupted. In such cases, an orthodontic appliance is fixed to the first molar. When the second molar has erupted, the orthodontic appliance is connected not only to the first molar but also to the adjoining teeth by tying them up with thin wires. This type of anchorage was also considered in the stress analysis.

Figure 4 shows an example of calculated stress distributions on the surface of the PDL when a force of $1.47 \mathrm{~N}$ was applied by a power chain (effective force of $1.08 \mathrm{~N}$ ) to a model that included the second molar. This figure shows the distributions of minimum principal stress at the external surface of the PDL on the pressurized side of each tooth root. The vertical axis denotes distance from the cervix to the apex of the tooth root. Since the canine and the second premolar have a simple root, the curves show the stress values at the nodes along the intersection of the external surface of the PDL and the mesiodistal vertical plane through the tooth axis. The compressive stress around the canine decreases rapidly from the cervix to the apex and turns into tensile stress 

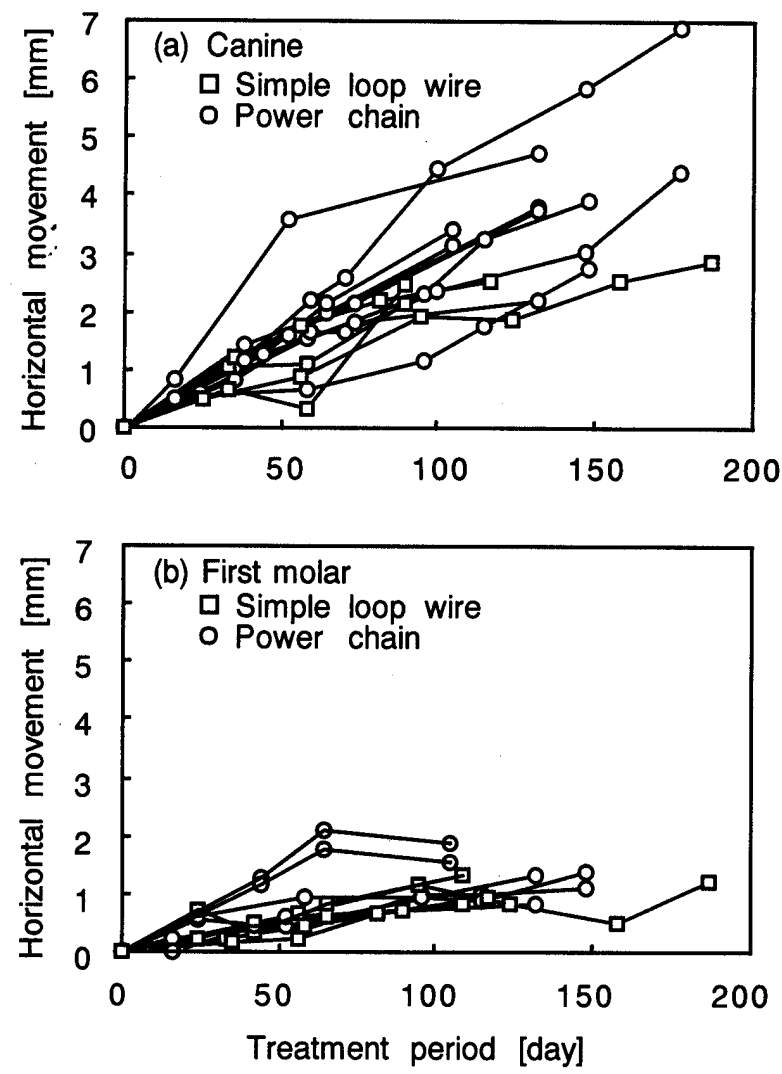

Fig. 5 Clinical measurements of horizontal tooth movement by $3-D$ shape measurement $(a)$ canine ( b ) first molar

because a point force was applied to the crown using the power chain. This stress inversion usually occurs in the apical third of the tooth as shown in this result. As the first and the second molars have a multiple root, the stress distributions around the individual root that showed the maximum compressive stress were evaluated. The stresses around the fixed teeth were much less than that around the canine because the applied force was shared by the teeth. Hereafter the first molars are analyzed as representative of the fixed tooth.

\subsection{Alveolar bone resorption}

Figure 5 shows the mesiodistal movements of the crown of the first molars and canines obtained from the clinical measurements mentioned in section 2.2 . The movements of the first molars were about $1 / 3$ those of the canines. The movements increased almost linearly with the number of days after treatment. Therefore, increases in the rate of movement per day for each tooth could be obtained from the slope of each regression line. The obtained rates of movement were then used to estimate the resorption rates of the alveolar bone to a unit stress.

\section{3 Alveolar bone resorption rate}

The relationship between minimum principal stress on the external surface of the PDL and alveolar

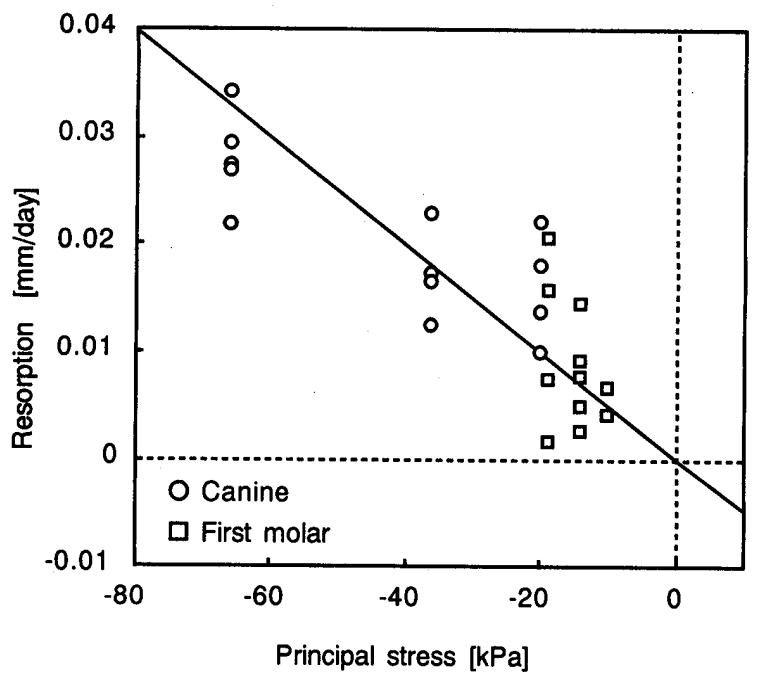

Fig. 6 Estimated relationship between principal stress around the tooth root and alveolar bone resorption during orthodontic treatment

bone resorption rate for the canine and the first molar are shown in Fig. 6. In our previous paper, we showed the relationship between stress distribution and corresponding alveolar bone resorption from the cervix to the apex of the canine ${ }^{(6)}$. In the present study, only the region of maximum compressive stress was analyzed to clarify whether there was a difference in the resorption rate between the canine and the first molar. In other words, stresses and resorptions at the node showing the maximum compressive stress and at the eight nodes adjacent to that node were selected and averaged to obtain a representative stress and resorption. A plot of these representative values is shown in Fig. 6. In this procedure, we supposed that tooth movement mainly depends on bone resorption in the region of the maximum compressive stress.

Resorption rates varied widely as shown in Fig. 6, but there was a clear tendency for the resorption rate to increase proportionally with the stress around the tooth root. Therefore, we calculated a regression line on the assumption that resorption does not occur at zero stress and obtained the alveolar bone resorption rate to a unit stress from the slope of the line. In spite of the great differences in stress and resorption between the canine and the first molar as described in the sections 3.1 and 3.2, there was no significant difference in the resorption rate obtained for the canine $(0.5$ micron $/(\mathrm{kPa} \cdot$ day $))$ and that obtained for the first molar $(0.7$ micron $/(\mathrm{kPa} \cdot \mathrm{day}))$. When calculating a regression line using the whole data, the slope of the line drawn was about 0.5 micron $/(\mathrm{kPa} \cdot$ day $)$ (Fig. 6).

\section{Discussions}

The relationship between stress around the tooth 
root and bone resorption was analyzed to estimate bone resorption rates of moved and fixed teeth. As was expected, the obtained resorption rates widely varied. This was likely due to differences between individuals, some assumptions ${ }^{(6)}$ made when calculating the resorption rate, geometrical differences between the finite element model and the subjects teeth, etc. Despite considerable errors in our analytical method, our approach to estimating the resorption rate to a unit stress at a tooth root is more directly connected with bone remodeling than conventional studies $^{(11)}$, which focus on the force-displacement relationship at the crown. Although the stress-movement relationship has been studied by several investigators, in all those studies the stress was estimated from the orthodontic force divided by a projected tooth root area on the assumption of constant stress distribution over the entire area. Lee ${ }^{(12)}$ reported that canine movement was 0.5 to $1 \mathrm{~mm} /$ week when the stress around canine roots estimated by the abovementioned method was 10 to $20 \mathrm{kPa}$. The bone resorption rate calculated from these values is 5 to 7 micron/ $(\mathrm{kPa} \cdot$ day). This rate is much larger than our result. Possible reasons for this difference are that the tooth movements reported by Lee were significantly greater than our measurements shown in Fig. 5( a ) ; that the tooth movements reported by Lee are those at the crown, whereas we transferred crown movements into root movements; and that a stress value estimated from the projected tooth root area is generally smaller than a peak stress value calculated from a $3-\mathrm{D}$ finite element analysis. The $3-\mathrm{D}$ analysis that focuses on the root, as attempted in this study, is essential for understanding a causal relation of orthodontic force and tooth movement.

As shown in Figs. 4 and 6, stress values around the first molar, that is a fixed tooth, were less than 1/ 3 those around the canine, because fixed teeth not only have a relatively large root area but because they also share the orthodontic force. Although the first molar must ideally be fixed as an anchorage, all of the first molars analyzed in this study were moved as shown in Fig. 5. There are no studies, to our knowledge, on the quantitative, simultaneous analysis of tooth movement for fixed and moved teeth; most studies at present focus on moved teeth. Moreover, we were also able to estimate the alveolar bone resorption rate to a unit stress in both moved and fixed teeth. We found no significant difference in the resorption rate between moved and fixed teeth as shown in Fig. 6 . This suggests that the movement of a tooth can be estimated from the stress distribution around the tooth root, regardless of species of teeth.

As described in the introduction, the basic con- cept of differential force that an optimal force acts to greater degree on moved teeth than on fixed teeth is widely accepted in orthodontics ${ }^{(1)}$. The basis of this concept is that there is a stress threshold for initiating bone resorption and an optimal stress for inducing the most efficient resorption. The force at the crown required to produce the optimal stress is called the optimal force; there have been many studies on this force since the first systematic study by Storey ${ }^{(11)}$. However, these studies were limited to the crown of tooth. The optimal stress and the stress threshold at the tooth root must be studied in connection with bone remodeling; however, the existence of these stresses has not yet been verified because an analytical method for estimating the alveolar bone resorption rate has not yet been established.

In our results, the stress values around fixed teeth were much smaller than those around moved teeth, resulting in almost proportionally less movement. In other words, no stress threshold or optimal stress were found in this study. However, this result does not preclude the concept of a differential force. The concept of differential force may be explained by differences in the geometry of the root of moved and fixed teeth. As for the optimal stress, the moved teeth analyzed in this study were treated adequately without the application of excessive orthodontic force, based on clinical experiences. It is well known that when an excessive force is applied to a tooth, the induction of osteoclasts is inhibited and the rate of bone resorption is decreased.

Further investigation through the application of our method to other orthodontic cases is needed to determine more accurately the relationship between stress and alveolar bone resorption. In order to verify the existence of optimal stress and differential force, the effects of these forces must also be studied at a cellular level. We have performed animal experiments and found that most of osteoclasts appear at localized sites where the compressive stress is within an appropriate range ${ }^{(8),(9)}$. A comparative study of animal experiments and clinical measurements will also clarify the quantitative relationship between mechanical stimuli and alveolar bone remodeling.

\section{Conclusions}

In this study, we estimated the resorption rate of alveolar bone to a unit stress in moved and fixed teeth on the basis of 3-D finite element analyses of teeth and the clinical measurements of 3-D tooth movement during orthodontic treatment. We found that an alveolar bone resorption rate to a unit stress is about 0.5 micron $/(\mathrm{kPa} \cdot$ day) and that there are no significant differences between the resorption rates of 
moved and fixed teeth. We also discussed these results in connection with differential force and concluded that differential force can be explained by differences in the geometry of the roots of moved and fixed teeth. However, the existence of an optimal stress and a stress threshold still needs to be verified through further investigation using quantitative analyses of clinical measurements and the results of animal experiments.

\section{Acknowledgement}

This study was supported in part by a Grant-inAid from the Ministry of Education, Science, Sports and Culture in Japan, and by the Hokkaido Foundation for the Promotion of Scientific and Industrial Technology.

\section{References}

(1) Burstone, C.J., The Biophysics of Bone Remodeling during Orthodontics -Optimal Force Considerations-. In the Biology of Tooth Movement, eds. by Norton, L.A. and Burstone, C.J., (1989), p. 321333, CRC Press (Boca Raton).

(2) Tanne, K., Sakuda, M. and Burstone, C.J., ThreeDimensional Finite Element Analysis for Stress in the Periodontal Tissue by Orthodontic Forces, Am. J. Orthod. Dentfac. Orthop., Vol. 92, No. 6 (1987), p. 499-505.

(3) Andersen, K.L., Mortensen, H.T., Pedersen, E.H. and Melsen, B., Determination of Stress Levels and Profiles in the Periodontal Ligament by Means of an Improved Three-Dimensional Finite Element Model for Various Types of Orthodontic and Natural Force System, J. Biomed. Eng., Vol. 13, No. 4 (1991), p. 293-303.

(4) Middleton, J., Jones, M.L. and Wilson, A.N., Three-Dimensional Analysis of Orthodontic Tooth Movement, J. Biomed. Eng., Vol. 12, No. 6 (1993), p. 319-327.
(5) Inoue, Y., Biomechanical Study on Orthodontic Tooth Movement by means of Numerical Simulation-Effects of Principal Stresses in Periodontal Membrane-, J. Osaka Univ. Dent. Soc., (in Japanese), Vol. 34, No. 2 (1989), p. 306-321.

(6) Morikawa, H., Nishihira, M., Yamamoto, K., Satoh, Y., Ishikawa, H. and Nakamura, S., Evaluation of Bone Resorption Rate during Alveolar Bone Remodeling by Orthodontic Treatment, Trans. Jpn. Soc. Mech. Eng., (in Japanese), Vol. 65, No. 589, C (1995), p. 3545-3551.

( 7 ) Nishikawa, H., Yamamoto, K., Mikami, T., Hayashi, S. and Nakamura, S., Measurement of Tooth Movement during Orthodontic Treatment and Model Analysis of Orthodontic Springs, IEICE Technical Report, (in Japanese), MBE88-26 (1988), p. 35-42.

(8) Yamamoto, K., Morikawa, H., Satoh, Y. and Nakamura, S., Computational Analysis of Bone Remodeling in Orthodontics. In Computational Biomechanics, eds. by Hayashi, K. and Ishikawa, H., (1996), p. 115-134, Springer-Verlag (Tokyo).

(9) Nishihira, M., Satoh, Y., Morikawa, H., Yamamoto, K., Ishikawa, H. and Nakamura, S., Stress Analysis and Osteoclast Appearance in Experimental Tooth Movement, J. Oromaxillofacial Biomech., (in Japanese), Vol.2, No. 2 (1996), p. 27-35.

(10) Yamamoto, K., Hayashi, S., Nishikawa, H., Nakamura, S. and Mikami, T., Measurement of Dental Cast Profile and Three-Dimensional Tooth Movemenl during Orthodontic Treatment, IEEE Trans. BME, Vol. 38, No. 4 (1991), p. 360-365.

(11) Storey, E. and Smith, R., Force in Orthodontics and Its Relation to Tooth Movement, Australian J. Dent., Vol. 56 (1952), p. 11-18.

(12) Lee, B.W., Relationship between Tooth-Movement Rate and Estimated Pressure Applied, J. Dent. Res., Vol. 44 (1965), p. 1053. 